\title{
Zur Hydrographie der Deutschen Bucht während des Winters 1962/63
}

\author{
ERICH GOEDECKE
}

Deutsches Hydrographisches Institut, Hamburg

\begin{abstract}
On the hydrography of the German Bight during the winter 1962/63. Very cold winters with severe ice conditions have hardly been investigated by hydrographers of German institutions. In order to assess the hydrographical situation during the cold winter $1962 / 63$, temperature and salinity information was used, provided by (a) five light vessels in the German Bight, (b) measurements obtained at several routine positions near Helgoland and along the route Hamburg-Hull, (c) data from the "Uthörn" cruises of the Biologische Anstalt Helgoland. Near the light vessels and at the Helgoland road, negative deviations of $3^{0}$ to $4^{\circ} \mathrm{C}$ were observed (monthly means). The corresponding salinity values are far above the normal. The severe ice winter ended approximately on the fifth of March, 1963. Temperature and salinity stratifications could even be observed during the coldest winter months. Vertical $\mathrm{T}^{\circ} \mathrm{C}$-distributions between Helgoland and the mouth of river Elbe demonstrate that the cooling of the sea water is not only advancing from east to west but also from the surface down to the bottom. Even at the time of the $\mathrm{T}^{\circ} \mathrm{C}$-minimum, there is a remarkable $\mathrm{T}^{\circ} \mathrm{C}$-difference between surface and bottom water. There are indications of strong mixing processes along the convergence of the German Bight.
\end{abstract}

\section{EINLEITUNG}

Seit Hunderten von Jahren sind die Temperaturen im mittleren Norddeutschland nicht so niedrig gewesen wie im Zeitraum zwischen Anfang März 1962 und Ende Februar 1963 (Scherhag 1963). Von vielen Autoren ist der Winter 1962/63 als "Jahrhundertwinter" oder ausgesprochener "Mitteleuropa-Winter" bezeichnet worden (v. Rudioff 1963). Selbst in England war es der kälteste Winter seit dem Jahre 1740 (RODEWALD 1963). Es erschien daher lohnend, sich mit den meteorologischen Faktoren zu beschäftigen, welche zu diesem außergewöhnlich kalten Winter 1962/63 geführt hatten. Insbesondere von englischer Seite ist zuerst auf die Auswirkungen hingewiesen worden, die dieser Winter auf die hydrographischen Verhältnisse in der Nordsee gehabt hat (ElLET'T 1963, TunNelx 1964). Innerhalb der Deutschen Bucht hat man sich nicht nur meteorologisch und hydrographisch, sondern auch biologisch mit den Auswirkungen der extremen Kältelage beschäftigt (KöтнKE \& Koops 1963; Forschungsfahrten der Biologischen Anstalt Helgoland). Uber die entsprechenden hydrographischen Verhältnisse in der westlichen Ostsee haben SINDERn (1963) und LORENZEN (1963) bereits berichtet. Im folgenden wird eine zusammenfassende Darstellung eines anläßlich des „Vierten Meeresbiologischen Symposions" in Hamburg gehaltenen 
Vortrages gegeben, dessen Originalinhalt an anderer Stelle veröffentlicht worden ist (GOEDECKE 1963).

\section{DIE MEERESKLIMATISCHEN VERHALTNISSE IN DER SÜDLICHEN NORDSEE}

Um das Ausmaß der hydrographischen Abweichungen vom Normalzustand verstehen zu können, ist es notwendig, sich vorweg mit dem winterlichen Meeresklima dieses Meeresgebietes anhand mittlerer Verhältnisse zu beschäftigen. Die Ergebnisse langjähriger meteorologisch-hydrographischer Untersuchungen haben ergeben, daß die hydrographischen Verhältnisse in bezug auf Luft- und Wassertemperatur, Salzgehalt und Strömungen sowie Wasserstand und Vereisung etc. in diesem Meeresgebiet von einem zum anderen Winter sehr verschieden sind (Goedecke 1957a-b, 1962a-b). Die folgende Ubersicht gibt eine Zusammenstellung derjenigen Winter, welche wegen ihrer starken Vereisung im deutschen Küstengebiet als außergewöhnlich zu bezeichnen sind:

$\begin{array}{llll}1921 / 22 & \text { eisreich } & 1941 / 42 & \text { sehr eisreich } \\ 1923 / 24 & \text { sehr eisreich } & 1946 / 47 & \text { extremer Eiswinter } \\ 1927 / 28 & \text { eisreich } & 1953 / 54 & \text { eisreich } \\ 1928 / 29 & \text { sehr eisreich } & 1954 / 55 & \text { eisreich } \\ 1939 / 40 & \text { sehr eisreich } & 1955 / 56 & \text { eisreich } \\ 1940 / 41 & \text { sehr eisreich } & 1962 / 63 & \text { sehr eisreich }\end{array}$

Es ist auffällig, daß in den 20er Jahren vier kalte bis sehr strenge Winter kurz hintereinander aufgetreten sind. Dagegen zeigen die 30er Jahre keinen derartigen eisreichen Winter. Nur der Winter 1936/37 kann in diesem Zusammenhang noch als kalt bezeichnet werden; in bezug auf die Stärke der Vereisung rangiert er in der Gruppe der mäßigen Eiswinter. In den 40er und 50er Jahren sind jeweils drei sehr kalte Winter dicht aufeinander gefolgt. Zu diesen außergewöhnlichen kalten Wintern gesellt sich noch derjenige von 1946/47, welcher in der gesamten Nord- und Ostsee heute noch eine meeresklimatische Ausnahmestellung einnimmt.

Wenn als Großwetterlagen die sogenannten Ostwetterlagen über Mittel- und Nordeuropa vorherrschen, so ist damit zu rechnen, daß im Winter eine verstärkte Abkïhlung des Meerwassers in der südöstlichen Nordsee erfolgt. Hierfür sind in erster Linie die stationären Hochdruckgebiete über Schweden und Finnland mit großräumiger Ausdehnung bis zum Europäischen Nordmeer und in zweiter Linie die ausgeprägten Hochdrucklagen ïber Ostrußland verantwortlich zu machen. Langdauernde Kaltlufteinbrüche aus nordöstlichen bis südöstlichen Richtungen führen zu einer bemerkenswerten Abkühlung des Meerwassers in den deutschen Küstengebieten der Nordsee. Die Abkühlung der Wassers in der Deutschen Bucht hängt nicht nur von der jeweiligen Höhe der Luftemperatur über diesem Meeresgebiet ab, sondern auch von morphologischen und dynamischen Faktoren. Hierzu gehören Wassertiefe, Wasserstand, Strömungen sowie Temperatur und Salzgehalt des Wassers. Einen maßgebenden Einfluß auf die winterliche Abkühlung des Meerwassers haben außerdem noch die Dauer, Häufig- 
keit und die jahreszeitliche Verteilung der Kälteperioden während der Wintermonate. Weiterhin ist der aufgespeicherte Wärmevorrat während der voraufgegangenen Monate von wesentlicher Bedeutung. Das Zusammenwirken all dieser Faktoren ist entscheidend für die Stärke der Abkühlung des Meerwassers und anschließend für diejenige der Vereisung pro Zeiteinheit in diesem Meeresgebiet.

Bevor wir uns mit den außergewöhnlichen hydrographischen Verhältnissen des Eiswinters $1962 / 63$ beschäftigen, ist es notwendig, sich einen Überblick über die mittleren hydrographischen Gegebenheiten während der Wintermonate zu verschaffen.

\section{MITTLERE HYDROGRAPHISCHE WINTERVERHALTNISSE IN DER DEUTSCHEN BUCHT}

Zwei charakteristische Wasserkörper beherrschen auf Grund ihres unterschiedlichen spezifischen Gewichtes die Deutsche Bucht: Salzreicheres Nordseewasser trifft mit salzärmerem Küstenwasser zusammen. Bekanntlich werden in der Nordsee die periodischen Gezeitenströme von den sogenannten unperiodischen Restströmen überlagert. Die Restströme verursachen die eigentlichen Wasserversetzungen. Mit ihnen werden spezifische hydrographische Eigenschaften und auch Meeresorganismen verfrachtet. Während des Winters strömt relativ warmes und salzreiches Nordseewasser in die Deutsche Bucht ein und setzt vorwiegend an der ostfriesischen Küste entlang nach Osten. Relativ kaltes und salzarmes Küstenwasser fließt in die offene Deutsche Bucht $a b$ und setzt vornehmlich an der nordfriesischen Küste entlang nach Norden und nordwestlich an Helgoland vorbei. Auf Grund der jeweils vorhandenen Dichteunterschiede stellt sich die Oberflächenzirkulation dieser beiden entgegengesetzt gerichteten Wasserkörper durch einen großräumigen zyklonalen Wirbel dar, welcher sich in der Vermischungszone (Konvergenz der Deutschen Bucht) in kleinere Teilwirbel aufteilt. Die wechselnde Größe der jahreszeitlichen Vermischung bestimmt den Habitus des Temperatur- und Salzgehaltsjahresganges.

Der mittlere Jahresgang von Temperatur und Salzgehalt verhält sich folgendermaßen: Sowohl im Oberflächen- als auch im Bodenwasser tritt das Jahresminimum vorwiegend im Februar auf. Das Bodenwasser ist im Februar durchschnittlich bis $0,5^{\circ} \mathrm{C}$ wärmer als die Oberfläche. Küstenwärts sinken die Februarmittel an der Oberfläche und am Boden gleichsinnig ab. Die nordfriesische Küstenregion und die Elbmündung sind stets kälter als die ostfriesische Küstenregion und die Wesermündung.

Ein gleichsinniger Verlauf des Salzgehaltsjahresganges ist bei den einzelnen Beobachtungsstationen untereinander nicht vorhanden. Er ist auch von Jahr zu Jahr verschieden. Die Extremwerte des Salzgehaltsjahresganges fallen mit denjenigen des Temperaturjahresganges zeitlich nicht zusammen. Die jahreszeitlichen Salzgehaltsschwankungen und somit auch die Eintrittszeiten der Extreme und ihre Höhe im Jahresgang werden gemeinsam durch die jeweilige Stärke des Nordsee-Einstromes und diejenige des Küstenabflusses in Abhängigkeit von meteorologischen Faktoren bedingt. Die Jahresextreme sind in der ostfriesischen Küstenregion und der Wesermündung stets höher als in der nordfriesischen Küstenregion und der Elbmündung. Die höchsten Salzgehalte 
treten vorwiegend in den Wintermonaten auf, wenn man einmal von den besonderen Verhältnissen in den Flußmündungen der Deutschen Bucht absieht.

Beim Vergleich des Salzgehaltsjahresganges mit dem der Wasserführung von Elbe und Weser besteht vor allem bei den küstennahen Beobachtungsstationen eine zeitliche Ubereinstimmung zwischen primären und sekundären Extremwerten. In bezug auf mittlere Verhältnisse kann man immer wieder feststellen, daß positive und negative Anomalien der Abflußmengen pro Zeiteinheit sich in den negativen und positiven Anomalien des Salzgehaltes widerspiegeln. Horizontal gesehen zeigen die Linien gleicher Eintrittszeiten der Salzgehaltsextreme im Oberflächenwasser der Deutschen Bucht eine deutliche Phasenverschiebung in Richtung Küste-See. Diese Phasenverschiebung ist im ostfriesischen Küstengebiet und vor den Flußmündungen sprunghaft ausgebildet, im nordfriesischen Küstengebiet jedoch weiträumig und stetig.

Diese hydrographischen Verhältnisse in bezug auf das Vorhandensein und die Stärke des Temperatur- und Salzgehaltsgradienten bedingen, daß die seewärtige Eisentwicklung an der ostfriesischen Seite gebremst, an der nordfriesischen Seite gefördert wird (GoEDECKE 1957a-b, 1958).

\section{ZUR HYDROGRAPHIE WAHREND DES WINTERS 1962/63}

Dem Verfasser standen die Temperatur- und Salzgehaltsbeobachtungen von fünf deutschen Nordsee-Feuerschiffen (GOEDECKE 1961/62), von zwei Terminstationen der Handelsschiff-Route Hamburg-Full (Goedecke 1962b) sowie von den Terminstatio-

Tabelle 1

Hydrographische Beobachtungsstationen

\begin{tabular}{|c|c|c|}
\hline Terminstationen & Geographische Lagebezeichnung & Institution \\
\hline $\begin{array}{l}\text { "Borkumriff" } \\
\text { "P 8“ } \\
\text { "P 12" } 12^{\circ} \\
\text { "Weser" } \\
\text { "Elbe 1" }\end{array}$ & $\begin{array}{l}\text { vor der Emsmündung } \\
\text { nordwestl. von Helgoland } \\
\text { südl. von Helgoland } \\
\text { vor der Wesermündung } \\
\text { vor der Elbemündung }\end{array}$ & \multirow{2}{*}{ 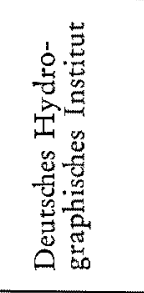 } \\
\hline $\begin{array}{l}\text { Hamburg/Hull Position I } \\
\text { Hamburg/Hull Position II }\end{array}$ & $\begin{array}{l}\text { bei Tonne P } 10 \text { (zwischen } \\
\text { Helgoland und F.Sch. "P 8“) } \\
\text { bei Tonne P } 5\end{array}$ & \\
\hline Helgoland-Reede & zwischen Insel und Düne & \multirow{3}{*}{ 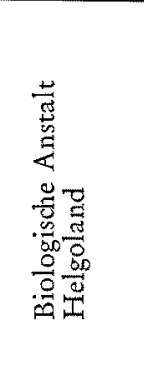 } \\
\hline $\begin{array}{l}\text { Station I (Hog Stean-Tonne) } \\
\text { Station II (früher IIa) } \\
\text { Station III (Tonne Sellebrunn) } \\
\text { Station IV (früher IIIa) } \\
\text { Station V (früher IIIb) } \\
\text { Station VI (früher IV) }\end{array}$ & $\begin{array}{l}\text { südl. von Helgoland } \\
\text { westl. von Helgoland } \\
\text { vor Nordeinfahrt Helgoländer Reede } \\
\text { in NNE } 5 \text { sm von Helgoland } \\
\text { bei Steingrund-Tonne östl. von Helgoland } \\
\text { bei Helgoland Tonne Düne } 02\end{array}$ & \\
\hline $\begin{array}{l}\text { Schnitt I } \\
\text { Schnitt II } \\
\text { Schnitt III } \\
\text { Schnitt IV }\end{array}$ & $\begin{array}{l}\text { Helgoland-Eidermündung } \\
\text { Helgoland-Elbemündung } \\
\text { Helgoland-Wesermündung } \\
\text { Helgoland-Feuerschiff „P } 8 \text { “ }\end{array}$ & \\
\hline
\end{tabular}


nen der Biologischen Anstalt Helgoland und den hydrographisch-biologischen Stationen ihrer besonderen Winterfahrten mit dem Forschungskutter "Uthörn" zur Verfügung. In Tabelle 1 wird eine Übersicht der Quellen des hydrographischen Beobachtungsmaterials für diesen außergewöhnlichen Winter in der Deutschen Bucht angegeben.

Die vorliegenden $\mathrm{T}^{\circ} \mathrm{C}$ - und $\mathrm{S} \% \mathrm{on}^{-B e o b a c h t u n g e n ~ l a s s e n ~ s i c h ~ i n ~ z w e i ~ g r o ß e ~ G r u p-~}$ pen zusammenfassen. In der einen Gruppe wird die Anderung der hydrographischen Situation an einem festen Punkt (Feuerschiff oder Terminstation) als Funktion der Zeit verfolgt. In der anderen Gruppe lassen sich Aussagen machen über die horizontale und vertikale Verteilung von Temperatur und Salzgehalt für bestimmte Perioden des Winters 1962/63. Entsprechende Diagramme und Horizontalkarten für das Oberflächenwasser und Vertikalschnitte für das Tiefenwasser sind entworfen und veröffentlicht worden (vgl. GoEDECKE 1963). Hier werden tabellarische Ubersichten gegeben, welche mit ihrem ausgewählten Zahlenmaterial biologischen Fragestellungen als Grundlage dienen mögen.

\section{Temperatur und Salzgehalt in der Deutschen Bucht}

In Tabelle 2 werden neben den Monatsmitteln die Temperatur- und Salzgehaltsanomalien (= Abweichungen vom langjährigen $\mathrm{T}^{0} \mathrm{C}$ - und $\mathrm{S} \% 0$-Monatsmittel) im Oberflächenwasser bei Helgoland-Reede und den deutschen Nordsee-Feuerschiffen für die Wintermonate Dezember 1962 bis April 1963 wiedergegeben. Für HelgolandReede sind als langjährige Mittelwerte diejenigen von $1927 / 44$ benutzt worden, für das Feuerschiff „Borkumriff“ gelten die Monatsmittel von 1954/60 und für die übrigen Feuerschiffe diejenigen von 1950/59 (GokDECKE 1962a). Die langjährigen Mittelwerte weisen das Temperaturminimum vorwiegend im Februar auf. Nur bei den weit westlich gelegenen Feuerschiffen „Borkumriff“ und „P 8 “ erscheint das mittlere Jahresminimum im März. Das korrespondierende Temperaturminimum liegt zwischen $2,5^{\circ}$ und $3,4^{\circ} \mathrm{C}$. Die Monatsmittel von 1962/63 dagegen liegen weit darunter, wie die Anomalien in Tabelle 2 deutlich zeigen. Es kommen im Durchschnitt negative Abweichungen von $3^{\circ}$ bis $4^{\circ} \mathrm{C}$ vor, maximal jedoch können sie fast $-5^{\circ} \mathrm{C}$ erreichen. Auch hier unterscheiden sich die thermischen Verhältnisse bei den küstennahen Stationen von denen der küstenfernen. Durchweg werden die stärksten negativen Abweichungen im Februar beobachtet, bei "Elbe $1^{\text {" }}$ aber schon im Januar. Sehr bemerkenswert ist noch die große negative Temperaturanomalie im April gegenüber derjenigen im Dezember 1962.

Bei den Salzgehaltsmonatsmitteln liegen die Verhältnisse vollkommen anders. Die langjährigen Mittelwerte weisen eine Salzgehaltsabnahme vom Winter zum Frühjahr hin auf. Im allgemeinen wird das S\%-Maximum in den Herbst- und Wintermonaten erreicht, das zugehörige Minimum dagegen im Frühjahr (April oder Mai), denn die starken Frühjahrs-Abflußmengen aus den großen Flußmündungsgebieten bewirken eine starke Salzgehaltsabnahme in der inneren Deutschen Bucht. Die Salzgehaltsmittel des Winters 1962/63 dagegen liegen aber weit darüber. Bei den küstennahen Terminstationen kommen positive Anomalien des mittleren Salzgehaltes von $1 \%$ on und mehr vor. Bei den küstenfernen Stationen liegen diese Werte nur wenig unterhalb dieser Grenze. 
Tabelle 2

T'emperatur- und Salzgehalts-Monatsmittel der Wintermonate 1962/63 und ihre Abweichungen (Abw.) vom langjährigen Mittel im Oberflächenwasser bei Helgoland-Reede und den Feuerschiffen

\begin{tabular}{|c|c|c|c|c|c|}
\hline Stationen & $\begin{array}{l}\text { Dezember } \\
1962 A b w .\end{array}$ & $\begin{array}{c}\text { Januar } \\
1963 A b r e .\end{array}$ & $\begin{array}{c}\text { Februar } \\
1963 \text { Abw. }\end{array}$ & $\begin{array}{c}\text { März } \\
1963 \text { Abw. }\end{array}$ & $\begin{array}{c}\text { April } \\
1963 \mathrm{Ab} w .\end{array}$ \\
\hline \multicolumn{6}{|c|}{ Temperatur $\left({ }^{\circ} \mathrm{C}\right)$} \\
\hline $\begin{array}{l}\text { Helgold. Reede } \\
\text { F.Sch. "P 8" } \\
\text { "Borkumriff" } \\
\text { "Weser" } \\
\text { F.Sh. "P 12" } \\
\text { "Elbe 1" }\end{array}$ & $\begin{array}{l}6,6-0,1 \\
6,8-0,9 \\
5,4-1,8 \\
5,2-1,2 \\
6,2-1,0 \\
4,6-1,9\end{array}$ & $\begin{array}{r}1,4-3,0 \\
2,4-3,0 \\
0,5-4,3 \\
0,3-3,5 \\
1,1-3,6 \\
-0,2-4,1\end{array}$ & $\begin{array}{l}-0,7-3,6 \\
-0,1-3,8 \\
-1,2-4,7 \\
-0,9-3,5 \\
-1,3-4,3 \\
-1,4-3,9\end{array}$ & $\begin{array}{l}+0,4-2,7 \\
+0,4-3,0 \\
-0,3-3,4 \\
+0,3-2,7 \\
-0,2-3,4 \\
+0,2-2,8\end{array}$ & $\begin{array}{c}3,0-2,2 \\
2,0-3,1 \\
3,6-1,9 \\
2,8-2,6 \\
(3,3)(-2,2) \\
3,2-2,4\end{array}$ \\
\hline \multicolumn{6}{|l|}{ Salzgehalt (S\%) } \\
\hline $\begin{array}{l}\text { Helgol. Reede } \\
\text { F.Sch. "P 8“ } \\
\text { "Borkumriff“ } \\
\text { "Weser“" } \\
\text { F.Sch. „P 12“ } \\
\text { "Elbe 1" }\end{array}$ & $\begin{array}{l}33,54+0,71 \\
33,76+0,11 \\
33,18+0,58 \\
32,84+0,66 \\
33,41+0,66 \\
32,45+0,71\end{array}$ & $\begin{array}{l}33,47+0,56 \\
33,94+0,31 \\
33,18+0,53 \\
32,80+1,20 \\
33,03+0,64 \\
31,87+0,63\end{array}$ & $\begin{array}{l}32,95+0,28 \\
33,60+0,01 \\
33,24+1,02 \\
32,35+0,63 \\
32,49+0,30 \\
31,78+0,70\end{array}$ & $\begin{array}{l}33,22+0,84 \\
33,88+0,56 \\
32,93+0,57 \\
32,89+0,93 \\
32,76+0,61 \\
31,70+0,85\end{array}$ & $\begin{array}{c}32,44+0,74 \\
33,90+0,64 \\
32,51+0,16 \\
32,73+0,78 \\
(33,27)(+1,95) \\
31,89+1,36\end{array}$ \\
\hline
\end{tabular}

Wie sind diese eigenartigen Salzgehaltsverhältnisse im Winter und Frühjahr zu verstehen, wenn man erfahrungsgemäß jedes Jahr damit rechnen kann, daß mit dem Beginn der anhaltenden Eisschmelze der Salzgehalt in der Deutschen Bucht wesentlich erniedrigt wird? Während der Zeit der Küstenvereisung ist praktisch gesehen kein merklicher Abfluß aus den Flußmündungstrichtern zu erwarten. Der Einstrom von Nordseewasser aus westlichen und nordwestlichen Richtungen in die innere Deutsche Bucht kann sich verstärken und für eine geraume Zeit anhalten. Der Salzgehalt in diesem Meeresgebiet wird zunehmen und nach einer gewissen Zeit einen konstanten Wert erreichen. Dieser Wert wird sich erst bei Eintritt der Eisschmelze wieder ändern, wenn in zunehmendem Maße der Küstenabfluß verstärkt einsetzen kann. Ausschlaggebend für dieses entgegengesetzte Verhalten des Oberflächensalzgehaltes war die auffallend geringe Niederschlagstätigkeit während der Monate Januar bis März. Im gesamten Gebiet der Bundesrepublik wurden im Januar 1963 nicht einmal 50\% der langährigen Mittelwerte erreicht. Die Wintermonate Februar und März waren zu trodken. Erst der April zeigte wieder nahezu normale Niederschlagsverhältnisse. Diese anomalen hydrometeorologischen Gegebenheiten im Bundesgebiet wie auch im weiteren Raum von Mitteleuropa haben im deutschen Küstengebiet der Nordsee bewirkt, daß der mittlere monatliche Abfluß der Elbe in der Zeit von Oktober 1962 bis März 1963 ein sehr bemerkenswertes Defizit aufweist, wenn man die Wasserstandsbeobachtungen vom Pegel Darchau an der Oberelbe berücksichtigt. In den Wintermonaten betragen die Istwerte der Abflußmengen nur 40 bis $50 \%$ der Sollwerte.

Außer der Betrachtung der mittleren langjährigen Monatswerte zusammen mit denen vom Winter 1962/63 sind für die Feuerschiffe "Elbe 1" und "P 8" sowie für Helgoland-Reede auch noch Dekadenmittel von Temperatur und Salzgehalt für diese Untersuchung herangezogen worden (vgl. Abb. 1-3 in Goedecke 1963). Als Beispiel werden in Tabelle 3 die entsprechenden Dekadenmittel von $\mathrm{T}^{\circ} \mathrm{C}$ und $\mathrm{S}^{\circ} \%$ im Ober- 
flächenwasser bei Helgoland-Reede angeführt. Es werden die korrespondierenden Werte vom Winter 1961/62 und 1962/63 miteinander in Vergleich gesetzt.

Auch hier ist eine große negative Abweichung der Wassertemperatur festzustellen. Nicht nur die langjährigen Monatsmittel, sondern auch schon die Dekadenmittel von einem zum anderen Winter weisen auf die außergewöhnliche Stellung des eisreichen

Tabelle 3

Dekadenmittel der Temperatur und des Salzgehaltes sowie deren Unterschiede im Oberflächenwasser bei Helgoland-Reede für die beiden Winter 1961/62 und 1962/63

\begin{tabular}{|lcrrrrrrr|}
\hline \multirow{2}{*}{ Monat } & Dekade & \multicolumn{3}{c}{ Temperatur $\left({ }^{\circ} \mathrm{C}\right)$} & \multicolumn{3}{c}{ Salzgehalt (S\%o) } \\
& & $1961 / 62$ & $1962 / 63$ & Diff. & $1961 / 62$ & $1962 / 63$ & Diff. \\
\hline Dezember & 1. & 8,4 & 7,9 & $-0,5$ & 32,97 & 33,66 & $+0,69$ \\
& 2. & 7,5 & 6,8 & $-0,7$ & 33,41 & 33,41 & $+0,00$ \\
& 3. & 6,0 & 5,2 & $-0,8$ & 33,51 & 33,65 & $+0,14$ \\
Januar & 1. & 4,9 & 3,0 & $-1,9$ & 32,93 & 33,87 & $+0,94$ \\
& 2. & 5,1 & 2,0 & $-3,1$ & 33,56 & 33,66 & $+0,10$ \\
& 3. & 4,1 & 0,3 & $-3,8$ & 32,08 & 33,18 & $+1,10$ \\
Februar & 1. & 4,0 & $-0,6$ & $-4,6$ & 32,28 & 32,90 & $+0,62$ \\
& 2. & 3,4 & $-0,8$ & $-4,2$ & 29,58 & 32,97 & $+3,39$ \\
März & 3. & 3,1 & $-0,6$ & $-3,7$ & 30,16 & 33,00 & $+2,84$ \\
& 1. & 2,8 & $-0,2$ & $-3,0$ & 31,48 & 33,06 & $+1,58$ \\
& 2. & 2,4 & $+0,4$ & $-2,0$ & 30,00 & 33,34 & $+3,34$ \\
April & 3. & 3,2 & $+0,9$ & $-2,3$ & 30,41 & 33,21 & $+2,80$ \\
& 1. & 4,1 & 2,0 & $-2,1$ & 31,95 & 31,74 & $-0,21$ \\
& 2. & 4,9 & 3,1 & $-1,8$ & 31,26 & 32,78 & $+1,52$ \\
& 3. & 6,5 & 4,0 & $-2,5$ & 30,07 & 32,95 & $+2,88$ \\
\hline
\end{tabular}

Winters 1962/63 hin. In den korrespondierenden Abbildungen (Abb. 2 u. 3, GoeDECKE 1963) kommt sehr schön zum Ausdruck, wie groß sich der thermische Einfluß von Küstennähe und Küstenferne bei den einzelnen Beobachtungsstationen auswirkt. Bei "Elbe 1 " setzt nämlich die stetige Abkühlung des Oberflächenwassers früher ein als bei „P 8 , ebenso ist die Erwärmung an der Oberfläche früher und rascher als bei „P 8“. Die zugehörigen S\%/00-Dekadenmittel in Tabelle 3 zeigen eindeutig, daß der Eiswinter 1962/63 mit seinen Salzgehaltswerten weit über denen vom vorigen Winter liegt. Geringer Niederschlag und Abfluß im Küstengebiet sowie auch der Umschwung der Großwetterlage vom "Hoch Mitteleuropa" auf „Süwest- und Westlagen" waren mit die Ursache, daß der mittlere Salzgehalt (ausgedrückt in Dekadenmittel) im großen und ganzen für die Jahreszeit zu hohe Werte aufwies. Mit dem Umschwung der Großwetterlage auf die sogenannten Westlagen ging eine Verstärkung der Oberflächenzirkulation in Richtung See-Küste vor sich. Der Einstrom von salzhaltigerem Nordseewasser in die innere Deutsche Bucht war wesentlich erhöht.

In der Meeresklimatologie ist es von jeher üblich, die hydrographischen Beobachtungen von Temperatur und Salzgehalt zu bestimmten Mittelwerten zusammenzufassen. Sind laufende tägliche Beobachtungen für ein ganzes Jahr vorhanden, wie etwa von den Feuerschiffen der Nordsee oder von der Terminstation Helgoland-Reede, so kann man diese Einzelmessungen, wenn eine gleichmäßige Verteilung der Beobachtun- 
gen für eine bestimmte Periode gewährleistet ist, zu Pentadenmitteln, Dekadenmitteln, Monatsmitteln und Jahresmitteln vereinigen. Liegen langjährige hydrographische Beobachtungen vor, so kann man neben diesen aufgeführten Mittelwerten auch noch langjährige Tagesmittel berechnen. Es ist seit langem in der Meeresklimatologie der Trend vorhanden, Jahres- und Monatsmittel aufzureißen und die hydrographischen Verhältnisse eines bestimmten Meeresgebietes mit Hilfe von Mittelwerten kürzerer Perioden darzustellen. Für bestimmte hydrographische und auch hydrobiologische Fragen sind neben den Mittelwerten bestimmter Periodenlänge die Einzelmessungen und Beobachtungen von ausschlaggebender Bedeutung. Derartige Einzelbeobachtungen von Tag zu Tag oder von Woche zu Woche sind in zwei Diagrammen dargestellt (vgl. Abb. 4 u. 5 in GOEDECKE 1963). Im ersten Fall wird durch die Gegenüberstellung von

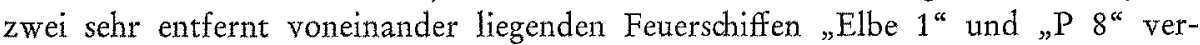
anschaulicht, wie die Größe der täglichen Abkühlung und Erwärmung im Winter und Frühjahr vonstatten geht. „Elbe $1^{\text {" }}$ als festlandnahe Beobadhtungsstation zeigt stets frühere und stärkere Abkühlung beziehungsweise Erwärmung als die küstenferne Beobachtungsstation „P 8“. Zum anderen wird gerade in solch einem Diagramm mit Einzelwerten gezeigt, wic eng die hydrographischen und meteorologischen Erscheinungen miteinander verkniupt sind. Nach Aufzeichnungen der monatlichen Witterungsberichte des Deutschen Wetterdienstes ist festzustellen, daß der sehr kalte und eisreiche Winter in Deutschland in allgemeinen um den 5. März 1963 sein Ende fand. Mit dem Umschwung der Großwetterlage zu diesem Zeitpunkt ist eine spontane Erwärmung des Oberflächenwassers in der Deutschen Bucht verbunden.

Im zweiten Fall (vgl. Abb. 5 in GoEDECKE 1963) wird der wöchentliche Verlauf von Temperatur und Salzgehalt im Oberflächen- und Bodenwasser bei Feuerschiff "Elbe 1" dargestellt. In der folgenden Tabelle 4 werden die Beobachtungen der hydrographischen Serien bei diesem Feuerschiff wiedergegeben, und zwar vom 1. Dezember 1962 bis zum 25. Mai 1963. Diese Beobachtungen stammen von $8 \mathrm{Uhr}$ und sind daher zu Zeiten mit verschieden starkem Gezeitenstrom vorgenommen worden. Tabelle 4 veranschaulicht sehr deutlich, daß auch in einem sehr kalten und eisreichen Winter die thermohaline Schichtung nicht aufgehoben zu sein braucht.

\section{Temperatur und Salzgebalt in der Tiefe zwischen Helgoland und Elbmïndung}

Tabelle 5 gibt die Temperatur- und Salzgehaltswerte für zwei Stationen wieder, welche Eckpunkte eines Vertikalschnittes repräsentieren, der yon GoEDECKE (1963, Abb. 6 bis 10) näher analysiert wurde. Am 20. November 1962 hat die küstenwärtige Abkühlung des Meerwassers besonders an der Oberfläche starke Fortschritte gemacht. Die Tiefe Rinne südlich von Helgoland (Station 1) zeigt noch relativ warmes Tiefenwasser von fast $10^{\circ} \mathrm{C}$. Im Übergangsgebiet (etwa bei Station 4) hat sich während der Sommer- und Herbstmonate zwischen Oberfläche und Boden nahe der Deckschicht eine thermohaline Sprungschicht herausgebildet, welche zu dieser Zeit noch gut erhalten ist. Durch dieses Ubergangsgebiet zieht sich die Vermischungszone der Deutschen Bucht, die sogenannte Konvergenz, nördlich von Helgoland hindurch. Der Salzgehalt nimmt bei Helgoland geringfügig $\mathrm{zu}$, von $33,60 \%$ an der Oberfläche auf $33,97 \%$ am Boden. 


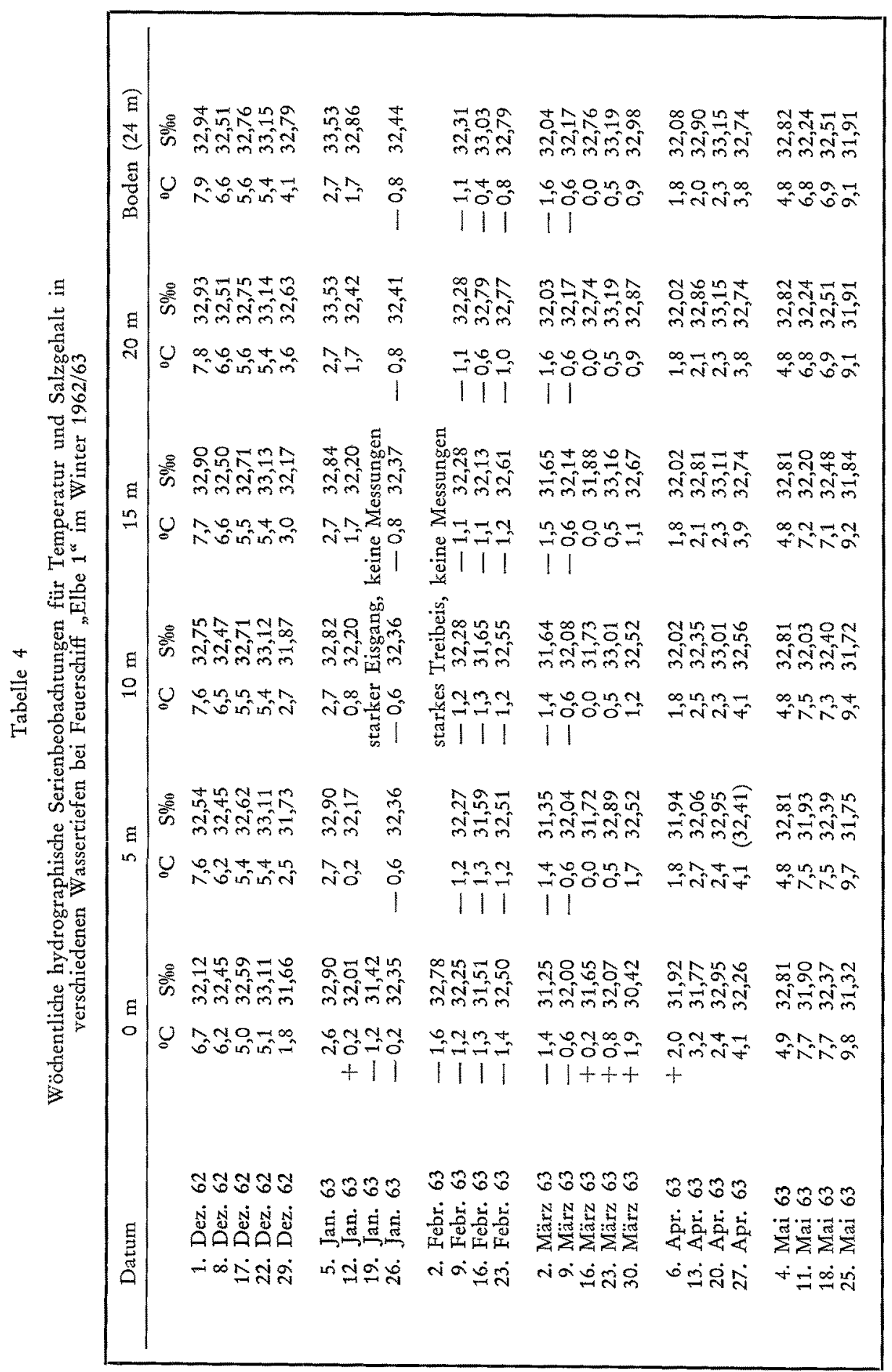


Bei Station 4 in der Elbmündung ist auf kürzerer Strecke eine stärkere Zunahme zu verzeichnen, von $30,45 \%$ in $0 \mathrm{~m}$ Tiefe auf $33,03 \%$ in $20 \mathrm{~m}$ Tiefe. Für den Monat Dezember sind keine entsprechenden Werte vorhanden, da der Forschungskutter der Biologischen Anstalt Helgoland wegen Werfliegezeit nicht zur Verfügung stand.

Am 29. 1. 1963 hat die Abkühlung des Meerwassers weiter zugenommen. Sie geht jetzt nicht nur vom Festland aus, sondern auch von einem in der Elbmündung vorhandenen Eisgürtel. Die Null-Grad-Isotherme liegt bereits dicht vor Helgoland. Weiterhin ist zu beachten, daß die Oberflächenschicht stets kälter ist als die Tiefenschicht.

Der Temperaturunterschied zwischen Oberfläche und Boden beträgt im Ostteil nur $0,1^{\circ} \mathrm{C}$, im Westteil aber $1,7^{\circ} \mathrm{C}$. Dieses Verhalten spricht dafür, daß die Abkühlung des Wassers nicht nur von Ost nach West, sondern auch von oben nach unten fortschreitet. Die Salzgehaltsverteilung zeigt nur geringe Unterschiede von oben nach unten. Die vertikale Temperaturverteilung am 12. Februar 1963 (vgl. Tab. 8 in GokDECKE 1963) kennzeichnet den Höhepunkt der Vereisung in der inneren Deutschen Bucht. Die Null-Grad-Isotherme liegt jetzt schon weit westlich von Helgoland. $Z_{\text {wi- }}$ schen Helgoland und der Elbmündung werden nur negative Wassertemperaturen beobachtet. Ein thermischer Unterschied zwischen der Deckschicht und der Bodenschicht ist immer noch vorhanden. Im Ostteil beträgt die Temperaturdifferenz etwa $0,2^{\circ} \mathrm{C}$ und im Westteil nur noch rund $0,6^{\circ} \mathrm{C}$. Die Abkihlung des Meerwassers erfaßt die gesamte Wassersäule von der Oberfläche bis zum Boden. Im Gegensatz zu den entsprechenden Verhältnissen in der südlichen und nördlichen Ostsee reicht die winterliche Konvektion von der Oberfläche bis zum Boden. Diese Vertikalströmungen dauern so lange, bis das Wasser bis zum Gefrierpunkt abgekühlt ist. Der Unterschied zwischen den thermischen Verhältnissen in der Nordsee und denen der Ostsee ist zu erklären erstens durch die Temperatur-Dichte-Funktion und zweitens durch die Funktion von Gefrierpunkt und Temperaturpunkt des Dichtemaximums des Meerwassers in Abhängigkeit vom Salzgehalt. Während die Temperatur-Dichte-Abhängigkeit bei normalen Flüssigkeiten nahezu linear verläuf, und zwar in dem Sinne, daß steigende Temperatur mit einer Dichteabnahme einhergeht, sind beim Wasser infolge seiner komplexen Zusammensetzung aus drei verschiedenartig aufgebauten Molekülpaketen besondere Verhältnisse vorhanden. In einem Temperatur-Dichte-Diagramm drückt sich diese Besonderheit so aus, daß die Temperatur-Dichte-Funktion einer parabelförmigen Kurve ähnelt. Bemerkenswert hierbei ist das Auftreten eines Dichtemaximums und dessen Verschiebung infolge steigenden Salzgehaltes zu Gebieten niedrigerer Temperatur. Im Meerwasser liegen im allgemeinen die hier gültigen Beziehungen so, daß mit steigendem Salzgehalt und abnehmender Temperatur die Dichte zunimmt und umgekehrt (KALLE 1945).

Eine ähnliche Verschiebung in gleicher Richtung bewirkt der steigende Salzgehalt auch bezüglich der Lage des Gefrierpunktes. Hier verschieben sich Gefrierpunkt und Temperaturpunkt des Dichtemaximums gemeinsam mit steigendem Salzgehalt in Richtung niedrigerer Temperaturen (KALLE 1949). Aber der Unterschied besteht darin, daß der Betrag der Verschiebung beim Gefrierpunkt etwa viermal geringer ist als beim Dichtemaximum. In einem Diagramm Dichtemaximum/Gefrierpunkt in Abhängigkeit von Temperatur und Salzgehalt werden sich beide Kurven überschneiden, und zwar etwa bei $25 \%$ Salzgehalt. Beim schwachsalzigen Meerwasser unter $25 \%$ liegt der 
Tabelle 5

Temperatur- und Salzgehalts-Serienbeobachtungen zwischen Helgoland (Station 1) und der Elbmïndung (Station 4) im Winter 1962/63

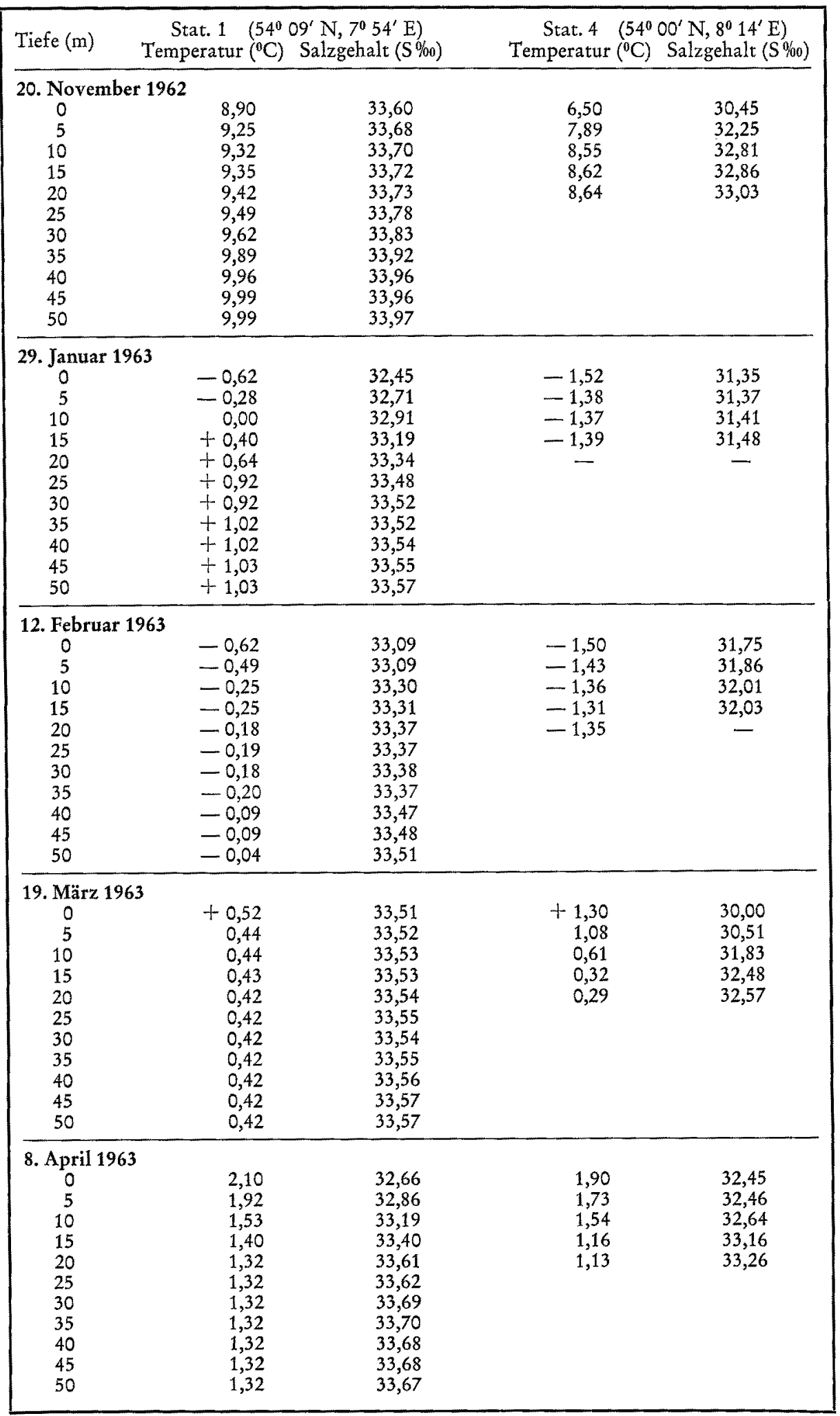


Gefrierpunkt tiefer als der Temperaturpunkt des Dichtemaximums. Beim ozeanischen Wasser von $35 \%$ ist es umgekehrt. Der Gefrierpunkt liegt bei rund $-2^{0} \mathrm{C}$ und der Temperaturpunkt des Dichtemaximums bei etwa $-3,5^{\circ} \mathrm{C}$. Gebiete mit Meerwasser unter $25 \%$ Salzgehalt gefrieren bedeutend schneller $\mathrm{zu}$ als diejenigen mit höherem Salzgehalt. Diese Überlegungen der Abhängigkeit von Gefrierpunkt und Dichte des Wassers kann man auch an dem Verhalten des Ostseewassers mit sehr niedrigem Salzgehalt und eines Süßßwassersees des Festlandes anstellen. Gerade in unserem Zusammenhang muß man besonders darauf hinweisen, wie langsam die Deutsche Bucht zufriert, ja daß die Nordsee iiberhaupt nicht vollkommen zufrieren kann, so lange nicht die gesamten Wassersäulen von der Oberfläche zum Boden bis zum Gefrierpunkt abgekühlt sind.

Betrachten wir nun wieder die hydrographischen Serien bei Helgoland und in der Elbmündung. Mit dem 19. März 1963 ist der eigentliche Eiswinter vorüber (Tab. 5). In der Deutschen Bucht bildet sich zuerst eine fast ausgeglichene und später eine umgekehrte Temperaturlage heraus (vgl. Abb. 9 in GoEDECKE 1963). Die Erwärmung des Wassers geht vom Festland aus und macht von Ost nach West weitere Fortschritte, wie die vertikale Temperaturverteilung in der Elbmündung zeigt. Zwischen Oberfläche und Boden besteht wiederum ein thermischer Unterschied. Dieser beträgt im Ostteil etwa $1,5^{\circ} \mathrm{C}$, im Westteil nur 0,10 $\mathrm{C}$. Dicht vor dem Elbmündungstrichter ist der Beginn einer stärkeren thermischen Sprungschichtbildung zu erkennen. In Tabelle 6 sind für die letzten beiden Stadien (19. März und 8. April 1963) die hydrographischen Serien bei dem Feuerschiff "Elbe 2 " aufgeführt, welche die vertikale Temperatur- und Salzgehaltsverteilung im Inneren der Elbmündung wiedergeben.

Infolge der Eisschmelze und der abfließenden Oberwassermengen der Elbe, auch wenn sie noch so gering sind, wird der Salzgehalt in der Elbmündung und der inneren

Tabelle 6

Temperatur- und Salzgehaltsbeobachtungen des F.K. "Uthörn“ beim Feuerschiff „Elbe 2" im März und April 1963

\begin{tabular}{|ccccc|}
\hline \multirow{2}{*}{ Tiefe $(\mathrm{m})$} & \multicolumn{2}{c|}{ 19. März 1963} & \multicolumn{2}{c|}{ 8. April 1963 } \\
& Temperatur $\left({ }^{\circ} \mathrm{C}\right)$ & Salzgehalt $(\% 0)$ & Temperatur ( $\left.{ }^{\circ} \mathrm{C}\right)$ & Salzgehalt (\%o) \\
\hline 0 & $+1,35$ & 25,01 & 2,51 & 28,38 \\
5 & 0,82 & 28,20 & 2,02 & 30,44 \\
10 & 0,70 & 29,70 & 1,43 & 32,35 \\
15 & 0,53 & 31,34 & 1,41 & 32,46 \\
20 & - & - & 1,39 & 32,47 \\
\hline
\end{tabular}

Deutschen Bucht herabgesetzt. Dabei kann sich zu dieser Zeit sogar eine etwas kräftigere haline Sprungschicht bilden (Tab. 6). Mit dem 8. April 1963 hat die Erwärmung des Wassers in der Deutschen Bucht bereits große ,Fortschritte gemacht (Tab. 5, 6). Die thermische Sprungschicht ist deutlich zu erkennen. Uber die zugehörigen Salzgehaltsverhältnisse ist zu sagen, daß sich vor und in der Flußmündung der Elbe eine stärker werdende haline Schichtung dicht unter der Oberfläche herausbildet. In der vertikalen Temperatur- und Salzgehaltsverteilung ist auf das Vorhandensein zweier Wasserkörper zu schließen. 


\section{Oberflächentemperaturen in der südlichen Deutschen Bucht}

In drei Karten ist die horizontale Temperaturverteilung an der Oberfläche in der südlichen Nordsee für bestimmte Perioden des Winters 1962/63 dargestellt worden (GOEDECKE 1963). In diesen Karten wird vor allem auf das Vorhandensein von zwei differenzierten Wasserkörpern der Deutschen Bucht hingewiesen, und zwar insofern, als gerade in solch einem kalten Winter der thermische Gegensatz zwischen der ostfriesischen und nordfriesischen Küstenregion herausgearbeitet werden kann. Während der ersten Periode, 23. bis 31. Januar 1963, trift relativ warmes Nordseewasser auf sehr kaltes Küstenwasser. Die großen Temperaturgradienten im Gebiet der Helgoländer Gewässer deuten auf stärkere Vermischungsvorgänge hin. Die Konvergenz der Deutschen Bucht ist in ihrer Wurzel zu erkennen. Bei diesen starken Vermischungsvorgängen, welche in der Zeit stärkster Abkühlung des Meerwassers stattfinden, kommt es vor, daß in der Helgoländer Bucht (oder auch westlich von Helgoland) Regionen relativ wärmeren Wassers von über $+1^{0} \mathrm{C}$ linsenförmig eingekreist werden. Diese Erscheinung ist nur dynamisch zu erklären. Mit der zunehmenden Abkühlung des Meerwassers seit September bis zum Jahresminimum bleibt im Zentralgebiet der südöstlichen Nordsee und der inneren Deutschen Bucht ein relativ warmer Wasserkörper so lange erhalten, bis er je nach Großwetterlage entweder zu einem kleinen Rest zusammengeschrumptt oder, wie in diesem Fall (siehe nächste Periode), zur Zeit des Höhepunktes der Vereisung völlig verschwunden ist. Die dynamische Ursache der Bildung solcher warmer Restwasserkörper ist in dem Vorhandensein eines Teilwirbels des großen zyklonalen Wirbels der Deutschen Bucht, welcher in der Konvergenz vorhanden ist, zu suchen (GoEDECKE 1941). Mit der zweiten Periode, 12. bis 28. Februar 1963, wird der Höhepunkt der Abkühlung beziehungsweise der Vereisung der Deutschen Bucht angezeigt. Die Null-Grad-Isotherme liegt weit westlich und läuft etwa beim Feuerschiff "P 8“ nach nordwestlicher Richtung aus (siehe die Abbildungen in GOEDECKE 1963). Die Konvergenz der Deutschen Bucht kommt infolge starker Vermischung der beiden verschiedenen Wasserkörper deutlich zum Ausdruck. Die Periode 2. bis 9. April 1963 zeigt den Fortschritt der Erwärmung der Deutschen Bucht. Sehr bemerkenswert in diesem Zusammenhang ist das Vorhandensein eines eingeschlossenen Kaltwasserkörpers entlang der ostfriesischen Küste. Dieser wird mit zunehmender Erwärmung von Osten und Westen her verkleinert und allmählich aufgehoben.

\section{ZUSAMMENFASSUNG}

1. Sehr kalte und sehr eisreiche Winter sind selten von deutschen Hydrographen untersucht worden. Das Studium der Temperatur- und Salzgehaltsverhältnisse während des Eiswinters 1962/63 in der südlichen Nordsee ist daher von besonderem Interesse. Die Beobachtungsdaten stammen von 5 deutschen Nordseefeuerschiffen, den Helgoländer und den Hamburg-Hull-Route Terminstationen sowie von den "Uthörn"-Fahrten der Biologischen Anstalt Helgoland.

2. Bei den Feuerschiffen und bei Helgoland-Reede sind negative monatliche Temperatur-Abweichungen von $3^{\circ}$ bis $4^{\circ} \mathrm{C}$ beobachtet worden. Die zugehörigen mittleren 
monatichen Salzgehaltsabweichungen liegen dagegen weit $\ddot{u} b$ e $r$ den Normalwerten.

3. Das Ende des Eiswinters wurde mit dem 5. März 1963 bestimmt. Eine Temperaturund Salzgehaltsschichtung blieb auch während der kältesten Wintermonate erhalten.

4. Die vertikale Temperaturverteilung zwischen Helgoland und der Elbmündung zeigt, daß die Abkühlung des Meerwassers nicht nur von Ost nach West fortschreitet, sondern auch von der Oberfläche zum Boden. Sogar zur Zeit des Temperaturminimums blieb eine bemerkenswerte Temperaturdifferenz zwischen der Oberfläche und dem Boden bestehen.

5. Die Anzeichen starker Vermischungsvorgänge entlang der Konvergenz der Deutschen Bucht werden dargestellt.

6. An Hand von Tabellen werden die wichtigsten Temperatur- und Salzgehaltsdaten zusammengestellt.

\section{ZITIERTE LITERATUR}

ElLETT, D. J., 1963. Surface temperature in the southern North Sea, January-March 1963. ICES Hydrogr. Comm., C. M. No 115.

GoedECKE, E., 1939a. Beitrag zur Hydrographie der Helgoland umgebenden Gewässer. 1. Die Oberflächenverhältnisse bei Helgoland-Reede. Annln Hydrogr. Berl. H. 4, 161-176.

- 1939b. -. 2. Die mittleren hydrographischen Verhältnisse bei den Helgoländer Terminstationen. Annln Hydrogr. Berl. H. 4, 224-234.

- 1940. Die heutige Kenntnis von der Morphologie und Hydrographie der Helgoländer Tiefen Rinne im Zusammenhang mit entsprechenden Verhältnissen der südöstlichen Deutschen Bucht. Annin Hydrogr. Berl. H. 12, 405-421.

- 1941. Beiträge zur Hydrographie der Konvergenz der Deutschen Bucht. Annln Hydrogr. Berl. H. 11, 345-362.

- 1957a. Das merkwürdige Verhalten der Eiswinter $1953 / 54$ bis $1955 / 56$. Seewart 18, 9-22; $50-65$.

- 1957b. Neuere Untersuchungen über die Vorbereitungs- und Abschmelzzeir des Eises in der Deutschen Bucht und westlichen Ostsee. Annln Met. 8, 80-92.

- 1958. Über Höhe und Eintrittszeit der Extreme sowie deren Schwankungen im mittleren Jahresgang von Temperatur, Salzgehalt und Dichte des Wassers in der Deutschen Bucht. Dt. bydrogr. Z. 11, 137-165.

- 1961/62. Uber die Bedeutung der deutschen Feuerschiffe als meereskundliche Beobachtungsstationen. Seewart 22, 196-210; 237-258, 23, 38.

- 1962a. Hydrographic conditions in the German Bight in 1960. Annls biol, Copenh. 17 (1960), 57-59.

- 1962b. Deutsche Handelsschiffe beobachten für den Internationalen Rat für Meeresforschung in Kopenhagen. Seewart 23, 149-163; 181-201.

- 1963. Uber die hydrographische Situation in der Deutschen Bucht während des Eiswinters 1962/63. Dt. bydrogr. Z. 16, 249-263.

Kalci, K., 1945. Der Stoffhaushalt des Meeres. In: Probleme der kosmischen Physik. Hrsg. von C. Jensen. Becker \& Erler, Leipzig, Bd. 23, 2. Aufl., 263 pp.

- 1949. Die natürlichen Eigenschaften der Gewässer. In: Handbuch d. Seefischerei Nordeuropas. Hrsg. von H. Lübbert (u. a.). Schweizerbart, Stuttgart, Bd. 1, H. 2, 37 pp.

Köтнке, H. \& Koops, H., 1963. Das Fischsterben in der Elbe im Winter 1962/63 - eine Folge der Verunreinigung des Elbwassers. Arch. FischWiss, 14, 50-56.

Lorenzen, J. M., 1963. Erfahrungen und Aufgaben des Eisbrecherwesens in der Ostsee. In: Ostseeschiffahrts- und Hafentage 1963 in Lübeck, 20.-22. Mai 1963, Der Senat der Hansestadt Lübeck, 68-83. 
Rodewald, M., 1963. Kältester Winter in England seit 1740. Wetterkarte Seewetteramt 11, Nr. 138.

Rudloff, H. v., 1963. Der Winter 1962/63 - ein Jahrhundertwinter! Witterungs-Scbnellber., Baden, Apr. 1963, Beil.

Scherhag, R., 1963. Die größte Kälteperiode seit 223 Jahren. Natwrw. Rdsch. 16, 169-174. Sindern, J., 1963. Temperatur- und Eisverhältnisse des Winters $1962 / 63$ in der westlichen Ostsee. Di. gewässerk. Mitt. 7, 154-159.

Tomczak, G. \& Goenecke, E., 1962. Monatskarten der Temperatur der Nordsee, dargestellt für verschiedene Tiefenhorizonte. Dt. bydrogr. Z., Erg. H., R. B., Nr. 7.

Tunnel, G. A., 1964. The winter of 1962-63 and its effect upon British coastal waters. Mar. Obs. 34, No 203, 21-32.

\section{Diskussion im Anschluß an den Vortrag GOEDECKE}

TомсZAк: Diese Untersuchungen zeigen, daß man offenbar mit wenig Oberflächenbeobachtungen ein gutes Bild davon erhalten kann, wie verschiedene Wasserkörper im Gebiet der Konvergenz gegeneinander verwirbeln. Ich möchte darauf hinweisen, daß ELLET im Seegebiet der Hoofden ähnliches gemacht hat. Die Ergebnisse der Arbeit ELLET sind als Sonderdruck beim Vorsitzenden einzusehen.

GOEDECKE: Ja, man muß sich wirklich wundern, aus welchem kleinen Material Herr ElLET es verstanden hat, sinnvolle Daten der Abkühlung und der Erwärmung in niederländischen und englischen Gewässern zu erarbeiten.

LüNEBURG: Es empfiehlt sich, zukünftig auch die Hydrographie der Eisscholle selbst zu erfassen.

Wellershauds: Welche hydrographischen Messungen wurden in der Ostsee während des strengen Winters vorgenommen?

KosLowsKI (zu WenLERShaus): Vier hydrographische Serien sind im Februar und März 1963 in der westlichen Ostsee durchgeführt worden, und zwar bei den Positionen der Feuerschiffe Fehmarnbelt und Kiel. 